\title{
Performance of Wireless Networks with Hidden Nodes: A Queuing-Theoretic Analysis*
}

\author{
Saikat Ray ${ }^{\mathrm{a} \dagger}$, David Starobinski ${ }^{\mathrm{a} \ddagger}$ and Jeffrey B. Carruthers ${ }^{\mathrm{a} \S}$

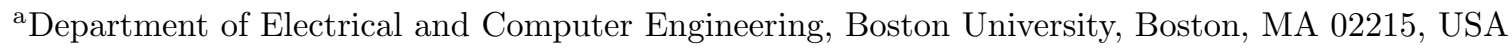

Hidden nodes are a fundamental problem that can potentially affect any wireless network where nodes cannot hear each other. Although the hidden node problem is well known, so far only few papers have quantified its effects in a comprehensive manner. This paper represents a first step towards getting a quantitative insight into the impact of hidden nodes on the performance of wireless networks. We first carry out an exact queuing-theoretic analysis for a 4-node segment and derive analytical expressions for the probability of packet collision, the mean packet delay, and the maximum throughput, based on a model that closely follows the IEEE 802.11 standard. We then extend the analysis and provide an approximation for a general linear topology that is asymptotically exact at low load. Finally, we perform detailed simulations to validate our analytical results and show their applicability to predict the performance of IEEE 802.11 networks with hidden nodes. The simulation and analysis closely match. Moreover, they reveal that the impact of hidden nodes propagates through the network causing some nodes to saturate at load as low as $15 \%$ of the capacity.

Keywords: IEEE 802.11, Hidden Nodes, Collision Probability, Delay Analysis, Maximum Throughput Analysis.

\section{Introduction}

In recent years, IEEE 802.11 standard Wireless Local Area Networks (WLANs) have rapidly gained popularity and become the solution of choice for networking homes and campuses [1]. These networks use a variant of Carrier Sense Multiple Access (CSMA) to control channel access by the nodes [2].

In CSMA, a node is allowed to transmit a packet only if it senses the channel idle. Packet collisions are rare as long as nodes can hear each other and the propagation delay is small. However, in wireless networks, nodes often cannot hear each other. Such networks are then likely to suffer from the so-called hidden node problem. This problem is illustrated in Fig. 1. In this topology, node $C$ cannot hear node $A$ 's transmissions. However, if node $C$ transmits while node

\footnotetext{
${ }^{*}$ This work was supported in part by the National Science Foundation under CAREER award ANI-0132802 and grant ANI-0240333 and by a SPRInG award from Boston University.

†Email: saikat@bu.edu

‡Email: staro@bu.edu

§Email: jbc@bu.edu
}

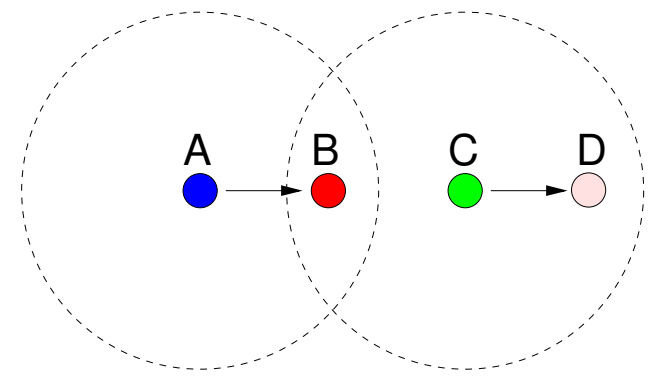

Figure 1. Packet collision due to a hidden node. If nodes $A$ and $C$ transmit simultaneously, node $A$ 's packet will collide.

$A$ is transmitting, node $A$ 's packet will collide. Node $C$ is the hidden node in this case. Note that this logical topology may in fact represent more complex networks; we provide an example in section 3.1.

Since hidden nodes cause packet collisions, their presence can severely affect the performance of wireless networks. As a matter of fact, the 
author of [3] considers the hidden node problem to be one of the "top ten challenges" in future wireless architectures. Although some hidden node mitigation techniques do exist, such as the RTS/CTS mechanism, they incur significant overhead and are often not used [4-6]. In the IEEE 802.11 standard, for instance, the $R T S$ / CTS mechanism is only optional. Thus, in practice, wireless networks are often exposed to the hidden node problem. As wireless networks proliferate, it is therefore vital to analyze and accurately quantify the impact of hidden nodes on network performance; not only to help understand the behavior of current networks, but also to help design future networks.

Although the hidden node problem is well known, few papers so far have been devoted to evaluating its impact on network performance. Instead, most previous works have focused on hidden node mitigation techniques, see [7] and references therein. We describe some of the main related work in section 2.2. In particular, we find that analytical models developed in previous work do not capture queuing and retransmission effects and oftentimes lead to inaccurate performance evaluation or trivial solutions (such as a single node capturing the channel).

This paper contributes to the fundamental understanding of how hidden nodes impact wireless networks. Our analytical models closely follow the IEEE 802.11 standard, and take into account queuing and retransmissions at each node. We first consider elementary 4-node topologies and derive exact expressions for (i) the probability of packet collision, (ii) the average packet delay, (iii) and the maximum throughput. We then extend the analysis to a general linear topology by iteratively applying the results obtained for the elementary topology. Although this extended analysis is provably exact only at low load, our simulations show that it remains fairly accurate over a wide range of load values. Using the NS simulator, we also show that our analysis predicts the performance of IEEE 802.11 networks with hidden nodes in a remarkably accurate fashion. Both the analysis and simulation reveal an interesting propagation effect which causes the nodes in the linear network to saturate at offered traffic load of as low as $15 \%$ of the (isolated) capacity, even though each node shares the channel with only one other node. This effect is reminiscent of influence-propagation phenomena observed in the context of multi-rate wireless networks [8].

This paper is organized as follows. In section 2, we provide the relevant background on the IEEE 802.11 protocol necessary for understanding this paper and discuss related work. In section 3, we describe our model and notation. As a motivation, we also present a simple analysis that ignores queuing and retransmission dynamics and show its limitation. Then, in section 4 we present the main results of this paper. In particular, analytical expressions for the probability of packet collision, maximum throughput, and mean packet delay for an elementary 4-node topology are provided in sections 4.1, 4.2 and 4.3, respectively, and an extension of these results to a general linear topology is presented in section 4.4. Several of the more technical proofs are deferred to section 5. We then present simulation results in section 6 , and conclude the paper in section 7 .

\section{Background}

\subsection{Outline of the IEEE 802.11 Basic Ac- cess Method}

In this section, we describe some of the salient features of the IEEE 802.11 Wireless LAN protocol that are most relevant to the rest of this paper. The protocol is described in detail in [1].

The IEEE 802.11 MAC protocol supports two types of access mode: Point Coordination Function (PCF) and Distributed Coordination Function (DCF). The DCF mode is more commonly used.

The DCF mode is essentially equivalent to CSMA. A node transmits a DATA packet if it senses the channel to be idle. The receiver, upon receiving an error-free packet, returns an $A c$ knowledgment $(A C K)$ packet. If no $A C K$ packet arrives in response to a $D A T A$ packet, the packet is retransmitted. The maximum allowed number of retransmissions is in general quite large. For instance, the default setting is 16 in Cisco aironet cards [9]. The retransmissions are separated by backoff intervals, which are integer multiples of 
BackoffSlotTime. The BackoffSlotTime, $20 \mu \mathrm{s}$, is a small fraction of the transmission time of a DATA packet (e.g. a 1500 byte packet takes about $12 \times 10^{3} \mu \mathrm{s}$ when transmitted at $1 \mathrm{Mb} / \mathrm{s}$ rate). Therefore, in most cases, an unsuccessful transmission is retransmitted within a short amount of time.

The DCF mode also supports the optional $R T S$ / CTS handshake that is designed to mitigate the hidden node problem. Since we are interested in quantifying the impact of hidden nodes in this paper, we will assume that nodes do not use this method, as it is often the case in practice.

\subsection{Related Work}

The CSMA protocol was introduced by Kleinrock and Tobagi in [2]. The authors also noted the hidden node problem, and proposed an alternate protocol, called the Busy Tone Multiple Access (BTMA) in [10]. In these papers, the analysis of the protocols has been carried out under the assumption that "the interarrival times of the point process defined by the start times of all the packets plus retransmissions are independent and exponentially distributed"; i.e. those form a Poisson process. This assumption is referred to as a "random-look" assumption since Poisson arrivals see time-averages (the so-called PASTA property) [11]. Many subsequent papers use the same assumption as a basis for their analysis, e.g. [1214] which propose various improvements over the basic CSMA/BTMA and [15] which point out the possible existence of hidden nodes in infrastructure mode WLAN.

The "random-look" assumption might be reasonable when the average retransmission delay is large compared to the packet transmission time. However, in the IEEE 802.11 standard, the retransmission delay is small compared to the packet transmission time. Thus, one should not expect such an assumption to provide a good approximation. To illustrate this point, we present in section 3.3 a simple analysis of the probability of a packet collision based on this "random-look" assumption and show that the result significantly underestimates the collision probability, even at low load.

In [16], the author studied the maximum throughput obtainable from an IEEE 802.11 network with no hidden node. The main modeling assumption is that every node always has a packet to transmit. The same assumption is used in $[17,18]$. We note, however, that this assumption can lead to trivial solutions when hidden nodes are present. For example, consider the topology shown in Fig. 1. In this topology, it is clear that if both nodes $A$ and $C$ always have packets to transmit, then node $C$ will always transmit, and node $A$ will be unable to transmit any packet. The techniques presented in [16], therefore, are not applicable in the present context. Other related works include $[19,20]$, which also do not take queuing into account.

In the context of ALOHA and CSMA/CD, early works carried out packet delay analysis. For example, Sidi and Segall considered a two user case in slotted ALOHA [21], and Takagi and Kleinrock analyzed a slotted CSMA/CD system [22]. Note that in the ALOHA protocol, retransmissions delays are large compared to the packet transmission time. In CSMA/CD, on the other hand, hidden nodes are not present since the medium is wired. More recently, authors in [23] carried out a queuing theoretical analysis of the Bluetooth system. Bluetooth, however, uses TDMA and therefore collisions due to hidden nodes is not an issue.

\section{Model and Simple Analysis}

Our goal is to analyze the performance of a wireless network with hidden nodes, taking into consideration queuing and retransmissions at each node. Since the analysis of a general network is difficult, we decompose the problem and consider first the simpler case of an elementary 4-node topology, which is interesting in its own sake. For this topology, we obtain exact expressions for the probability of packet collision, average system delay, and maximum throughput. Using an iterative technique, we then extend these results to a general linear topology and provide approximations that are asymptotically exact at low load. 


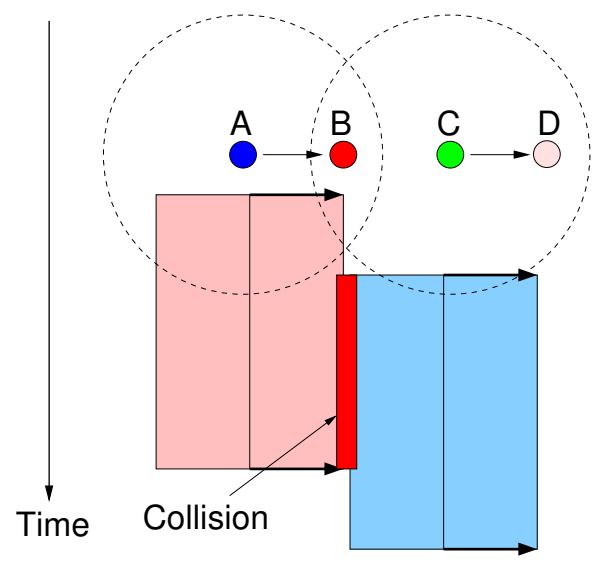

(a) Logical Topology

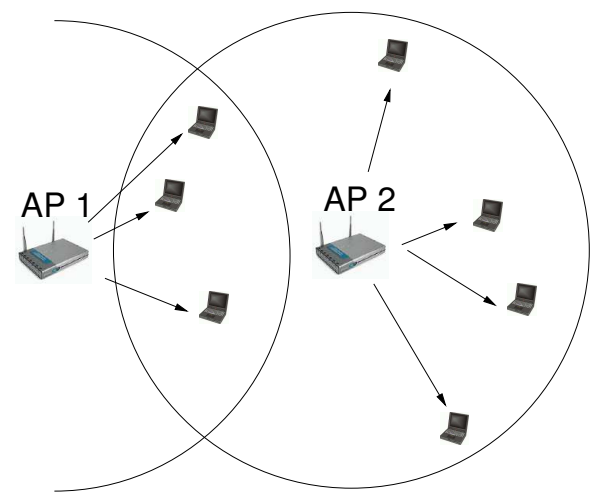

(b) Physical layout

Figure 2. Packet collision due to a hidden node. During the time node $A$ transmits a packet, node $C$ senses the channel to be idle and starts transmitting a packet to node $D$. This transmission collides with node $A$ 's transmission.

\subsection{The Elementary Topology}

In this section, we describe our model of the elementary 4-node topology. The elementary topology we consider is shown in Fig. 2 (this is a detailed version of Fig. 1). In this topology, node $A$ sends packets to node $B$ and node $C$ sends packets to node $D$. We consider omni-directional transmissions; the transmission ranges of nodes $A$ and $C$ are depicted by the circles. As shown in the figure, nodes $A$ and $C$ cannot hear each other; node $B$ hears both nodes $A$ and $C$; node $D$ hears only node $C$. The channel is noise-free, so that packets are lost only because of collisions. Each node uses CSMA, and propagation delay is negligible.

It is easy to see that node $A$ 's transmissions experience collisions due to the hidden node $C$. An example is shown in Fig. 2(a). During the time node $A$ is transmitting, node $C$ finds the channel idle and begins transmitting its own packet to node $D$. This transmission also reaches node $B$ and destroys node $A$ 's packet. On the other hand, node $C$ 's packets are always received by node $D$.

It is worth pointing out that this topology models well some network configurations of practical interest. Fig. 2(b) shows an example. In this figure, there are two overlapping IEEE 802.11 standard infrastructure network operating in the same channel (which happens in commercial buildings when multiple businesses install separate WLANs compelling some networks to share the same channel as there are only three nonoverlapping channels). The access points - denoted by "AP 1" and "AP 2" - do not hear each other. Moreover, under usual usage pattern, the uplink traffic (client to AP) in each network can be neglected compared to the downlink traffic. Then, this physical configuration is logically equivalent to the topology shown in Fig. 2(a): AP 1 is represented by node $A$, and AP 2 by node $C$. The client nodes are represented by nodes $B$ and $D$ respectively. Therefore, it is interesting to analyze this topology for its own sake.

We purposely assume that node $C$ does not send packets to node $B$; otherwise the throughput 
would be close to zero even at low load. The reason is that any packet collision at node $B$ triggers retransmissions by both nodes $A$ and $C$. These retransmissions repeatedly collide resulting in a vanishing network throughput. Thus, under any reasonable base-station selection algorithm, the node-AP associations will eventually converge to the logical topology shown in Fig. 2(a) [24].

\subsection{Statistical Model}

We now introduce our notation and statistical model for the analysis. Both nodes $A$ and $C$ maintain infinite buffer queues. The queues at nodes $A$ and $C$ are denoted by $Q_{A}$ and $Q_{C}$ respectively. The exogenous arrivals to $Q_{A}$ and $Q_{C}$ follow independent Poisson processes with rate $\lambda_{A}$ (number of packets per unit of time) and $\lambda_{C}$ respectively. The DATA packet size is fixed and the transmission time of each packet is $T$. The transmission time of $A C K$ packets is negligible. The assumptions of Poisson arrivals and fixed size packets lend to analytical tractability. These assumptions are, however, not unrealistic. For example, if the nodes $A$ and $C$ are access points, as shown in Fig. 2(b), then the traffic consists of many independent streams which is well modeled by Poisson arrivals.

It is worth noting that the assumption that the input process to the queue corresponds to a Poisson process does not imply that packet transmissions and retransmissions, which form the output process, also follow a Poisson process. In fact, proper characterization of the output process is the main challenge to accurately predict quantities of interest, such as delay and maximum throughput. The analysis that we perform in this paper addresses this challenge.

We denote the offered traffic load at node $A$ by $\rho_{\mathrm{A}}=\lambda_{A} T$ and at node $C$ by $\rho_{\mathrm{C}}=\lambda_{C} T$. For guaranteeing the stability of the system, it is necessary that $\rho_{\mathrm{A}}<1$ and $\rho_{\mathrm{C}}<1$. These conditions are usually not sufficient because they do not consider the time spent on retransmissions. We assume that the system is started at time $t=-\infty$, so that it reaches its steady state at time $t=0$. The steady-state number of packets in $Q_{A}$ and $Q_{C}$ is denoted by the random variables $q_{A}$ and $q_{C}$ respectively.
In the configuration considered, node $C$ 's packets are all correctly received at node $D$ and, thus, never retransmitted. Moreover, node $C$ is always free to transmit. Therefore, the statistical behavior of node $C$ corresponds to an $M / D / 1$ queue with service time $T$. The distribution of $q_{C}$ is therefore well known (see, for instance, [25, page 220]).

However, a packet sent by node $A$ collides if it overlaps with node $C$ 's transmission. Thus, node $A$ has to retransmit unsuccessful packets. So, in spite of Poisson arrivals of fixed length packets, the behavior of node $A$ does not correspond to an $M / D / 1$ queue. We model packet retransmissions in the following way: (i) the time between retransmissions (i.e., the backoff delay) is negligible and (ii) the maximum number of retransmissions allowed for each packet is unlimited. Our analysis presented in the section 4 is exact under these two assumptions. These modeling assumptions are not very restrictive in practice. Indeed, the analysis shows that the average number of retransmissions per packet is smaller than 1.5 as long as the queues are stable. Furthermore, in section 6.2 , we carry out detailed simulation of an IEEE 802.11 standard network with the NS simulator [26]. Simulation results confirm that our analytical models are able to accurately predict the performance of an IEEE 802.11 standard network.

\subsection{A Simple "Random-Look" Analysis}

As a motivation to our exact analysis, described in section 4 , we now carry out a simple approximate analysis based on the "random-look" assumption and show its limitation.

Without any loss of generality, let the time at which node $A$ starts a packet transmission be $t=0$. This packet could either be the initial transmission attempt, or a retransmission. Our goal is to compute the probability that this packet collides at the receiver, i.e., node $B$. For this purpose, we condition on the state of node $C$ at time $t=0$. Let the queue length at node $C$ at time $t=0$ be denoted by the random variable $q_{C}(0)$. We distinguish between the cases where $q_{C}(0)=0$, and $q_{C}(0)>0$. We thus have

$$
\operatorname{Pr}\{\text { Collision }\}
$$




$$
\begin{aligned}
& =\operatorname{Pr}\left\{\text { Collision } \mid q_{C}(0)>0\right\} \cdot \operatorname{Pr}\left\{q_{C}(0)>0\right\} \\
& +\operatorname{Pr}\left\{\text { Collision } \mid q_{C}(0)=0\right\} \cdot \operatorname{Pr}\left\{q_{C}(0)=0\right\} .
\end{aligned}
$$

The conditional probabilities are easy to compute. If $q_{C}(0)>0$, then node $C$ is currently transmitting. Therefore, node $A$ 's packet collides with probability 1, i.e., $\operatorname{Pr}\left\{\right.$ Collision $\left.\mid q_{C}(0)>0\right\}=1$. On the other hand, if $q_{C}(0)=0$, i.e. if $Q_{C}$ is empty at $t=0$, a collision will happen only if a packet arrives at node $C$ before $t=T$ (note that this packet is immediately transmitted). Since the arrival process to node $C$ is Poisson with rate $\lambda_{C}$, we get $\operatorname{Pr}\left\{\right.$ Collision $\left.\mid q_{C}(0)=0\right\}=1-e^{-\rho_{\mathrm{C}}}$.

To compute $\operatorname{Pr}\left\{q_{C}(0)=0\right\}$ and its complement, we now make use of the "random-look" assumption, i.e., that the state of node $C$ 's queue at the time when node $A$ starts a packet transmission is the same as at any random point of time [27]. Based on this assumption, we have $\operatorname{Pr}\left\{q_{C}(0)=0\right\}=\operatorname{Pr}\left\{q_{C}=0\right\}=1-\rho_{\mathrm{C}}$ (we remind the reader that $q_{C}$ is a random variable that corresponds to the steady-state queue length whereas $q_{C}(0)$ is a random variable denoting the queue length at time $t=0$ ). Therefore, we obtain the following expression for the collision probability in the hidden node case:

$$
\begin{aligned}
\operatorname{Pr}\{\text { Collision }\} & =1 \cdot \rho_{\mathrm{C}}+\left(1-e^{-\rho_{\mathrm{C}}}\right)\left(1-\rho_{\mathrm{C}}\right) \\
& =1-e^{-\rho_{\mathrm{C}}}\left(1-\rho_{\mathrm{C}}\right) .
\end{aligned}
$$

To illustrate the issues with this analysis at the very outset, Fig. 3 compares the analytical prediction made by Eq. 2 with simulation results for this system. The simulation set-up is described in detail in section 6 .

Figure 3 shows that the simple analysis significantly underestimates the collision probability, which may in turn lead to incorrect packet delay estimation. Moreover, it is clear from the figure that the slope of the simulated and theoretical curves differ as $\rho \rightarrow 0$. Therefore, the analysis is not asymptotically exact as $\rho \rightarrow 0 .^{5}$ One of the

\footnotetext{
${ }^{5}$ We define an analysis asymptotically exact as $\rho \rightarrow 0$ if the analytical function $f(\rho)$ and the exact function $g(\rho)$ satisfy $\lim _{\rho \rightarrow 0} f(\rho) / g(\rho)=1$. If $f(0)=g(0)=0$, then the definition implies that $f^{\prime}(0)=g^{\prime}(0)$.
}

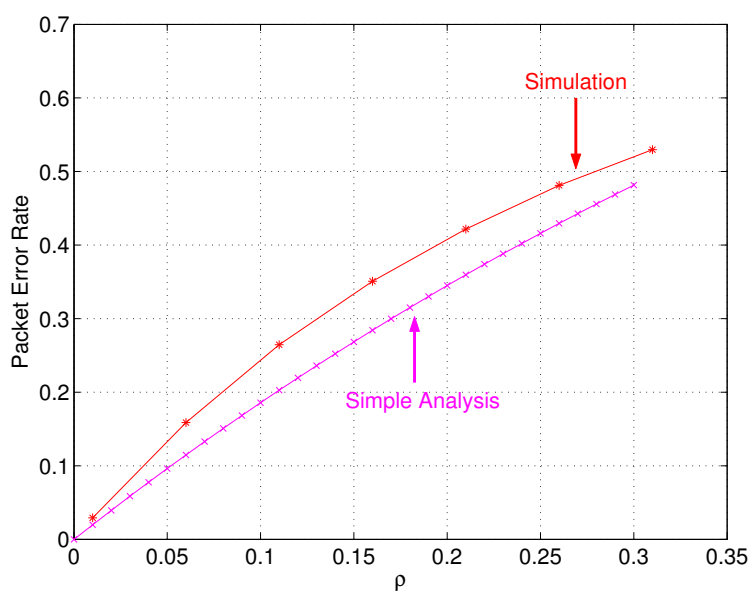

Figure 3. Probability of packet collision. Comparison between the simulation outcome and the predictions of the simple analysis.

reasons for the discrepancy is that when a DATA packet sent by node $A$ collides, it is retransmitted after a short backoff time period and this retransmission has a higher chance of colliding again since node $C$ may not yet have completed its transmission. So, the random-look assumption does not provide a good quality approximation, even at low load.

\section{Analysis and Main Results}

In this section, we present our main analytical results. In particular, we derive the probability of collision, maximum throughput and mean packet delay for the elementary topology in sections 4.1, 4.2 and 4.3, respectively, and generalize these results for a general linear topology in section 4.4. Some of the more lengthy proofs needed for the derivation are deferred to section 5 .

\subsection{Probability of Packet Collision in the Elementary Topology}

Without loss of generality, suppose node $A$ starts the transmission of a new (fresh) packet at time $t=0$. If node $\mathrm{C}$ does not transmit anytime 
during the interval $[0, T]$, then node $A$ 's transmission will be successful. Otherwise, node $A$ needs to retransmit its packet. The retransmission will take place during the interval $[T, 2 T]$ (we remind that the backoff delay is assumed to be negligible). Again, this transmission will be successful only if node $C$ does not transmit during that interval, and so forth. In general, node $A$ will require $N$ attempts until it succeeds in transmitting its packet, where $N$ is the smallest positive integer such that node $C$ does not transmit during the whole interval $[(N-1) T, N T]$ (i.e. the queue at node $C$ remains empty).

Let $E[N]$ denote the mean number of transmissions per packet. The packet collision probability, denoted by $P_{\text {coll }}$, corresponds to the fraction of unsuccessful transmissions, that is,

$P_{\text {coll }}=\frac{E[N]-1}{E[N]}=1-\frac{1}{E[N]}$.

Therefore, the derivation of an expression for $P_{\text {coll }}$ reduces to computing $E[N]$.

Let $q_{C}(t)$ denote the queue length distribution of node $C$ at time $t$. The derivation of $E[N]$ is performed by conditioning on the following two possible states of node $C$ at time $t=0$ : (a) $q_{C}(0)=0$, i.e., node $C$ does not transmit, or (b) $q_{C}(0)>0$, i.e., node $C$ does transmit. We therefore have

$$
\begin{aligned}
E[N] & =E\left[N \mid q_{C}(0)>0\right) \cdot \operatorname{Pr}\left\{q_{C}(0)>0\right\} \\
& +E\left[N \mid q_{C}(0)=0\right) \cdot \operatorname{Pr}\left\{q_{C}(0)=0\right\}
\end{aligned}
$$

Define the constant $\kappa=1+\frac{W_{0}\left(-\rho_{\mathrm{C}} e^{-\rho_{\mathrm{A}}-\rho_{\mathrm{C}}}\right)}{\rho_{\mathrm{C}}}$. Here $W_{0}(z)$ denotes the Lambert function, which is the solution to the equation $x e^{x}=z$ in the unknown variable $x$ [28]. In sections 5.1, 5.2 and 5.3, we will show that:

$$
\begin{gathered}
E\left[N \mid q_{C}(0)=0\right]=\frac{e^{\rho_{\mathrm{C}}}}{1-\rho_{\mathrm{C}}} \\
E\left[N \mid q_{C}(0)>0\right]= \\
\frac{\rho_{\mathrm{A}}+\kappa \rho_{\mathrm{C}}+\kappa \rho_{\mathrm{A}} e^{\rho_{\mathrm{C}}}+\frac{\kappa \rho_{\mathrm{A}}}{e^{\rho_{\mathrm{A}}-1}}}{\kappa \rho_{\mathrm{A}}\left(1-\rho_{\mathrm{C}}\right)} \\
\operatorname{Pr}\left\{q_{C}(0)>0\right\}= \\
\frac{\left(e^{\rho_{\mathrm{A}}}-1\right)\left(e^{\rho_{\mathrm{C}}}-P_{\text {coll }}-e^{\rho_{\mathrm{C}}} P_{\text {coll }}\right)}{1-P_{\text {coll }}}
\end{gathered}
$$

The expression for $\operatorname{Pr}\left\{q_{C}(0)=0\right\}$ is given by the complementary probability of Eq. 7. Note that $\operatorname{Pr}\left\{q_{C}(0)=0\right\} \neq \operatorname{Pr}\left\{q_{C}=0\right\}$ since $q_{C}$ denotes the steady-state value, whereas $q_{C}(0)$ denotes the queue length at a special instant, namely when node $A$ starts transmitting a packet (note also the difference in notation).

An expression for $E[N]$ in terms of $P_{\text {coll }}$ is obtained by substituting Eqs. 5, 6, and 7 into Eq. 4 . Substituting $E[N]$ into the L.H.S of Equation 3 yields an equation in $P_{\text {coll }}$. The solution is

$$
P_{\text {coll }}=1-\frac{\left(e^{\rho_{\mathrm{A}}}-1\right)-\frac{\kappa \rho_{\mathrm{A}} \rho_{\mathrm{C}}}{\rho_{\mathrm{A}}+\kappa \rho_{\mathrm{C}}}}{\left(e^{\rho_{\mathrm{A}}}-1\right)\left(e^{\rho_{\mathrm{C}}}+\frac{\rho_{\mathrm{C}}}{\rho_{\mathrm{A}}}\right)-\frac{\kappa \rho_{\mathrm{C}}}{\rho_{\mathrm{A}}+\kappa \rho_{\mathrm{C}}}},
$$

which is the desired expression for the probability of packet collision.

\subsection{Maximum Throughput in the Elemen- tary Topology}

The maximum throughput of the network is defined to be the largest traffic load at which the expected queue lengths at nodes $A$ and $C$ remain finite. The maximum throughput is therefore the boundary of the stable region. In the stable region, the following two conditions must hold: (i) $\operatorname{Pr}\left\{q_{A}=0\right\}>0$, and (ii) $\operatorname{Pr}\left\{q_{C}=0\right\}>0$. Since $Q_{C}$ is an $M / D / 1$ queue, $\operatorname{Pr}\left\{q_{C}=0\right\}=1-\rho_{\mathrm{C}}$, whence $\rho_{\mathrm{C}}$ must be smaller than 1 for condition (i) to hold. We use Little's law to find the $\operatorname{Pr}\left\{q_{A}=0\right\}$. Since, on average a packet at node $A$ takes $E[N]=\frac{1}{1-P_{\text {coll }}}$ attempts for successful transmission, the utilization of $Q_{A}$ is given by $\overline{\rho_{\mathrm{A}}}=\lambda_{A} T E[N]$. Then, the probability that $Q_{A}$ is empty is given by Little's Law:

$\operatorname{Pr}\left\{q_{A}=0\right\}=1-\overline{\rho_{\mathrm{A}}}=1-\frac{\rho_{\mathrm{A}}}{1-P_{\text {coll }}}$.

So, for condition (ii) to hold, $\rho_{\mathrm{A}}$ must be smaller than $1-P_{\text {coll }}$, where $P_{\text {coll }}$ is given by Eq. 8 .

Eq. 9 does not yield a closed-form formula, but allows us to compute the maximum throughput numerically. For example, with $\rho_{\mathrm{A}}=\rho_{\mathrm{C}}=\rho$, condition (ii) numerically translates to $\rho<0.401$ by using Eq. 9 . Since $\rho<0.401$ also satisfies condition (i), we find that the maximum throughput is $\rho^{*}=0.401$.

It is interesting to note that with $\rho_{\mathrm{A}}=\rho_{\mathrm{C}}=\rho$ the maximum throughput is lower than $\rho=0.5$. 
The general linear case is even more surprising; for some nodes, the saturation occurs at load $\rho=0.15$ even though each node shares the channel with only one other node. We discuss this result in section 4.4 .

Finally, we note that with $\rho<0.401$, the average number of retransmissions per packet is always smaller than 1.5. Thus, our assumption that the maximum allowed number of retransmissions is unlimited is not very restrictive in practice.

\subsection{Mean Packet Delay in the Elementary Topology}

In this section, we derive the average system time spent by a packet at node $A$ (or, simply the delay). Due to space constraint, we will only sketch the outline.

Let $N$ attempts be required to transmit a fresh packet successfully. Then, the service time of this packet is $N T$. We note that the service times of successive packets are independent, but not identically distributed. In particular, as shown in sections 5.1 and 5.2, the expected service time depends on the state of node $C$ at the beginning of a fresh packet transmission. Therefore, the Pollaczek-Khinchin (P-K) mean value formula [11, page 187] is not applicable directly. Instead, we derive the average system time in a manner similar to that done in [29, page 188].

Let $T_{Q}$ be the average waiting time in queue, and $T_{\text {sys }}$ the average system time, including both queuing and service. Clearly,

$T_{\text {sys }}=T_{Q}+T E[N]=T_{Q}+T /\left(1-P_{\text {coll }}\right)$.

Now, let $N_{Q}$ be the mean number of packets waiting in queue (but not in service) found by a new arrival. Then, using Little's law, $N_{Q}=\lambda_{A} T_{Q}$. Also, let $R$ be the mean residual service time of the packet in service if sampled at a random time. Then,

$$
\begin{aligned}
T_{Q}= & N_{Q} T E\left[N \mid q_{C}(0)=0\right] \\
& +R\left(1-\operatorname{Pr}\left\{q_{A}=0\right\}\right) \\
= & \lambda_{A} T_{Q} T E\left[N \mid q_{C}(0)=0\right] \\
& +R\left(1-\operatorname{Pr}\left\{q_{A}=0\right\}\right) .
\end{aligned}
$$

The conditioning in the expectation term follows from the fact that $q_{C}(0)=0$ for all packets that are enqueued, as explained in section 5.3. The mean residual service time $R$ is given by the following [11, page 172]

$$
\begin{aligned}
R & =\frac{E\left[(N T)^{2} \mid q_{C}(0)=0\right] \operatorname{Pr}\left\{q_{C}(0)=0\right\}}{2 T E[N]} \\
& +\frac{E\left[(N T)^{2} \mid q_{C}(0)>0\right] \operatorname{Pr}\left\{q_{C}(0)>0\right\}}{2 T E[N]} .
\end{aligned}
$$

The second moment computations are long, but similar to the procedures described in sections 5.1 and 5.2. The final expression for the case $\rho_{\mathrm{A}}=$ $\rho_{\mathrm{C}}=\rho$ turns out to be the expression shown in Eq. 14, which is the desired average packet delay.

\subsection{Extension to General Linear Network}

In this section, we extend our results of the elementary topology to a general linear topology. The linear topology is shown in Fig. 4. The nodes are arranged in pairs, $\left(A_{i}, B_{i}\right)(i=0,1, \ldots)$. Each node can sense/interfere with only its nearest neighbors. Node $A_{i}$ sends packets to node $B_{i}$; exogenous arrivals to each node $A_{i}$ follow an independent Poisson process with rate $\lambda_{i}$. As explained in section $3.1, B_{i}$ could represent a set of nodes instead of a single node.

The packet transmissions from node $A_{i}$ experience a probability of packet collision $P_{i}$. Our goal is to approximate $P_{i}(i=0,1, \ldots)$ using the result obtained in section 4.1. We do this by iteratively calculating $P_{i}$ 's, starting from $P_{0}$.

Since $A_{0}$ is the only node which can send packets to $B_{0}$, packets sent by node $A_{0}$ do not experience any collision, and $P_{0}=0$. Moreover, since $P_{0}=0$, the topology formed by the four nodes $A_{1}, B_{1}, A_{0}, B_{0}$ is the same as the elementary topology depicted in Fig. 2(a). Therefore, $P_{1}$ is given by Eq. 8, with $\rho_{\mathrm{A}} \leftarrow \rho_{1}=\lambda_{1} T$ and $\rho_{\mathrm{C}} \leftarrow \rho_{0}=\lambda_{0} T$.

Now, in the general case, assume $P_{i}$ has been computed. Then, the effective load on node $A_{i}$ is equal to $\bar{\rho}_{i}=\frac{\lambda_{i} T}{1-P_{i}}$. Also, the busy periods of node $A_{i}(i=0,1,2, \ldots)$ are still integer multiples of $T$. So, the behavior of node $A_{i}$ can be approximated by an $M / D / 1$ queue with rate $\bar{\rho}_{i}$. Therefore, an expression for $P_{i+1}$ can be derived using Eq. 8, with $\rho_{\mathrm{A}} \leftarrow \rho_{i+1}=\lambda_{i+1} T$ and $\rho_{\mathrm{C}} \leftarrow \bar{\rho}_{i}$. This iterative procedure can then be used to compute $P_{i}$ for all $i, i=0,1,2, \ldots$. We note that 


$$
\begin{aligned}
T_{\text {Sys }} / T= & \frac{-2-4 \kappa-\rho+2 \rho(\kappa+\rho)-e^{3 \rho}(1+\kappa)(2-\rho)(1-2 \rho)}{2\left(e^{\rho}-1\right)(1-\rho)\left(1-\rho-\rho e^{\rho}\right)\left(1+\kappa-e^{\rho}(1+\kappa)+\rho \kappa\right)}+ \\
& \frac{e^{2 \rho}(1+\kappa)(2+\rho(-9+2 \rho))+e^{\rho}\left(2+\rho(5-2 \rho)+\kappa\left(4+6 \rho^{2}-4 \rho^{3}\right)\right)}{2\left(e^{\rho}-1\right)(1-\rho)\left(1-\rho-\rho e^{\rho}\right)\left(1+\kappa-e^{\rho}(1+\kappa)+\rho \kappa\right)} \\
& \bullet \mathrm{A}_{2} \longrightarrow \mathrm{B}_{2} \mathrm{~A}_{1} \longrightarrow \mathrm{B}_{1} \mathrm{~A}_{0}
\end{aligned}
$$
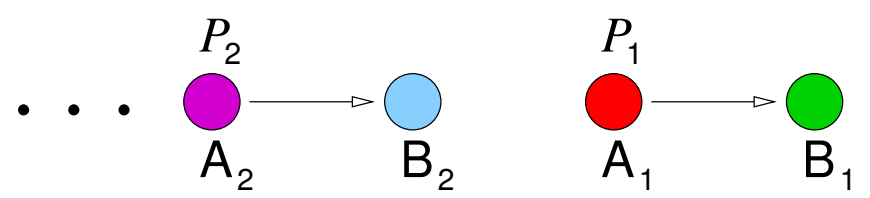

Figure 4. General linear network.

the $M / D / 1$ approximation is asymptotically exact at low load, as the number of retransmissions tends to zero in this regime. Our simulation results in section 6 show that this approximation carries over to fairly large load values.

It is interesting to note that the procedure generalizes to the case when the network is infinite. In particular, assume that $\rho_{i}=\rho$ for all $i$, and the sequence, $P_{0}, P_{1}, P_{2}, \ldots$ converges to $P$. Then, $P$ is the probability of collision experienced by the nodes $A_{n}(n \geq N)$, with $N$ sufficiently large ${ }^{6}$. Then, $P$ is given by substituting $\rho_{\mathrm{A}} \leftarrow \rho$ and $\rho_{\mathrm{C}} \leftarrow \frac{\rho}{1-P}$ in Eq. 8, and solving the resultant fixed point equation.

\section{Proof of Key Expressions}

\subsection{Calculation of $E\left[N \mid q_{C}(0)=0\right]$}

In this section, we derive Eq. 5. First, we recall that $Q_{C}$ behaves as an $M / D / 1$ queue. This queue passes through alternate cycles of idle and busy periods. Let us refer to Fig. 5, which shows the fashion in which idle periods alternate with busy periods. We denote the idle period durations by $X_{1}, X_{2}, X_{3}, \ldots$ and the busy period durations by $L_{1}, L_{2}, L_{3}, \ldots$, etc. $X_{1}$ is measured from $t=0$. Idle periods are i.i.d. exponentially distributed random variables with mean $E[X]=1 / \lambda_{C}$, and

\footnotetext{
${ }^{6}$ More precisely, $P_{n}$ is arbitrarily close to $P$; i.e. given $\epsilon>0,\left|P-P_{n}\right|<\epsilon$ for all $n \geq N$.
}

busy periods are i.i.d random variables with mean $E[L]=T /\left(1-\rho_{\mathrm{C}}\right)([11]$, Chapter 5.8). We note that the duration of each busy period is an integer multiple of $T$, since the service time (the transmission time) of each packet is deterministic and equal to $T$.

Next, we recall that node $A$ succeeds in transmitting its packet at the $N$-th attempt, that is, during the interval $[(N-1) T, N T]$. Let us denote by $M$, the number of busy periods of $Q_{C}$ until node $A$ successfully transmits its packet (in Fig. $5, M=3$ ). We note that the end of the $M$-th busy period of $Q_{C}$ always falls within the interval $[(N-2) T,(N-1) T]$. Next, denote by $Y$ the time interval between the end of the $M$-th busy period of $Q_{C}$ and the point of time $t=(N-1) T$. $Y$ is defined to be 0 if the first transmission is successful, i.e. $N=1$. The variables $N, M$, and $Y$ are all random. The relation between these random variables can be expressed as follows

$N T=\sum_{i=1}^{M} X_{i}+\sum_{i=1}^{M} L_{i}+Y+T$.

By taking the expectation on both sides of Eq. 15 and dividing by $T$, we obtain

$$
\begin{aligned}
& E\left[N \mid q_{C}(0)=0\right] \\
= & \frac{1}{T} E\left[\sum_{i=1}^{M} X_{i}\right]+\frac{1}{T} E\left[\sum_{i=1}^{M} L_{i}\right]+\frac{1}{T} E[Y]+1
\end{aligned}
$$




$$
\begin{aligned}
& =\frac{1}{T} E\left[\sum_{i=1}^{M} X_{i}+Y\right]+\frac{1}{T} E\left[E\left[\sum_{i=1}^{M} L_{i} \mid M\right]\right]+1 \\
& =\frac{1}{T} E\left[\sum_{i=1}^{M} X_{i}+Y\right]+E[M] \frac{E[L]}{T}+1 \\
& =\frac{1}{T} E\left[\sum_{i=1}^{M} X_{i}+Y\right]+E[M] \frac{1}{\left(1-\rho_{\mathrm{C}}\right)}+1
\end{aligned}
$$

The transition from Eq. 16 to Eq. 17 follows from the fact that the busy periods of $Q_{C}$ are i.i.d. and independent of $M$.

Our goal now is to derive expressions for $E\left[\sum_{i=1}^{M} X_{i}+Y\right]$ and $E[M]$. The key observation for deriving these quantities is that the expression $\sum_{i=1}^{M} X_{i}+Y$ must be an integer multiple of $T$. This property follows from Eq. 15 and the fact that the sum of the durations of busy periods, i.e. $\sum_{i=1}^{M} L_{i}$, is itself an integer multiple of $T$. We therefore can write

$\sum_{i=1}^{M} X_{i}+Y=(K-1) T$,

where $K \geq 1$ is an integer. Our next step is to compute $E[K]$ which, from Eq. 19, will allow us to determine $E\left[\sum_{i=1}^{M} X_{i}+Y\right]$.

We proceed in the following way. Construct a new time axis $t^{\prime}$ from $t$ by removing the busy periods $L_{i}$ 's. This new axis is shown in Fig. 5. As shown in this figure, the $m$-th busy period on $t^{\prime}$ axis is represented by a point occurrence at time $t^{\prime}=\sum_{i=1}^{m} X_{i}$. These occurrences are separated by i.i.d exponential variables, $X_{i}$ 's. So, they constitute a Poisson process with intensity $\lambda_{C}$. We denote this process by $\mathcal{P}$.

Furthermore, since each busy period is an integer multiple of $T$, the interval $[(N-1) T, N T]$ on $t$ axis is mapped to $[(K-1) T, K T]$ on $t^{\prime}$ axis. $K$ is therefore characterized to be the smallest positive integer so that the interval $[(K-1) T, K T]$ on $t^{\prime}$ axis has no arrival of the process $\mathcal{P}$.

Now we are in a position to compute $E[K]$. Since $\mathcal{P}$ is a Poisson process with rate $\lambda_{C}$, the probability that there is at least one arrival of $\mathcal{P}$ during any interval on length $T$ on $t^{\prime}$ axis is
$\left(1-e^{-\rho_{\mathrm{C}}}\right)$. Thus,

$$
\begin{aligned}
\operatorname{Pr}(K=k) & =\left(1-e^{-\rho_{\mathrm{C}}}\right)^{k-1} e^{-\rho_{\mathrm{C}}}, \text { and } \\
E[K] & =e^{\rho_{\mathrm{C}}} .
\end{aligned}
$$

$E[M]$ is also calculated with ease using the properties of $\mathcal{P}$. On $t^{\prime}$ axis, $M$ is simply the total number of arrivals of $\mathcal{P}$ in the interval $[0, K T]$. Define $\nu_{j}$ to be the number of arrivals of the process $\mathcal{P}$ during the interval $[(j-1) T, j T]$ on $t^{\prime}$ axis. For example, in Fig. $5, \nu_{1}=2$ and $\nu_{2}=1$. Then, $M$ is the sum of arrivals in each of the sub-intervals, i.e.

$$
M=\sum_{j=1}^{K-1} \nu_{j} .
$$

The sum runs up to $(K-1)$ since $\nu_{K}=0$ by the definition of $K$. By taking expectation on both sides, we get

$$
\begin{aligned}
& E[M] \\
= & (E[K]-1) E[\nu \mid \nu>0] \\
= & \left(e^{\rho_{\mathrm{C}}}-1\right) \frac{\rho_{\mathrm{C}}}{1-e^{-\rho_{\mathrm{C}}}}=\rho_{\mathrm{C}} e^{\rho_{\mathrm{C}}},
\end{aligned}
$$

where we omit the subscript of $\nu$ as they are i.i.d.

Substituting $E[K]$ from Eq. 21 and $E[M]$ from Eq. 24 in Eq. 18, we get

$$
\begin{aligned}
& E\left[N \mid q_{C}(0)=0\right] \\
= & \frac{1}{T} E\left[\sum_{i=1}^{M} X_{i}+Y\right]+E[M] \frac{1}{\left(1-\rho_{\mathrm{C}}\right)}+1 \\
= & {\left[e^{\rho_{\mathrm{C}}}-1\right]+\left[\rho_{\mathrm{C}} e^{\rho_{\mathrm{C}}}\right] \frac{1}{\left(1-\rho_{\mathrm{C}}\right)}+1 } \\
= & \frac{e^{\rho_{\mathrm{C}}}}{1-\rho_{\mathrm{C}}}
\end{aligned}
$$

This proves Eq. 5 .

We now compute a quantity that will be used in section 5.3. Let $W$ be the amount of time node $A$ transmits and node $C$ does not during the transmission attempts of this packet. Then,

$W=\left[\sum_{i=1}^{M} X_{i}+Y\right]+T$.

Therefore,

$$
E\left[W \mid q_{C}(0)=0\right]=E\left[\sum_{i=1}^{M} X_{i}+Y\right]+T
$$




$$
=T\left[e^{\rho_{\mathrm{C}}}-1\right]+T=T e^{\rho_{\mathrm{C}}} .
$$

5.2. Calculation of $E\left[N \mid q_{C}(0)>0\right]$

Now we derive Eq. 6. The derivation is similar to the derivation of Eq. 5 presented in section 5.1 except that we need to consider an additional residual busy period of $Q_{C}$.

Let us refer to Fig. 6 . It is given that $Q_{C}$ is going through a busy period at time $t=0$. Let this busy period end at $t=L_{0}$. Now, similar to section 5.1 , define $L_{i}(i=1,2, \ldots)$ to be the length of $i$-th busy period, $X_{i}$ to be the idle period preceding $i$-th busy period, and $M$ to be the number of busy periods of $Q_{C}$ following $t=L_{0}$ until node $A$ successfully transmits its packet during the interval $[(N-1) T, N T]$. The time interval between the end of the $M$-th busy period of $Q_{C}$ and the point of time $t=(N-1) T$ is again denoted by $Y$. These random quantities are related by the following equation,

$N T=\sum_{i=1}^{M} X_{i}+\sum_{i=1}^{M} L_{i}+Y+T+L_{0}$.

We take expectations on both sides of Eq. 30 and obtain

$$
\begin{aligned}
& E\left[N \mid q_{C}(0)>0\right] \\
= & \frac{1}{T} E\left[\sum_{i=1}^{M} X_{i}+Y\right]+\frac{1}{T} E\left[E\left[\sum_{i=1}^{M} L_{i} \mid M\right]\right] \\
& +1+\frac{1}{T} E\left[L_{0}\right] \\
= & \frac{1}{T} E\left[\sum_{i=1}^{M} X_{i}+Y\right]+E[M] \frac{E[L]}{T}+1 \\
& +\frac{1}{T} E\left[L_{0}\right] .
\end{aligned}
$$

Our goal now is to obtain the individual expectations. We begin by $E\left[L_{0}\right]$.

First note that, although $L_{0}$ is the residual busy period of $Q_{C}, t=0$ is not a random instant. Thus, the standard expression for the residual time does not apply. We will therefore compute $E\left[L_{0}\right]$ from the basic principles.

Figure 7 shows a typical sample path for the case $q_{C}(0)>0$. During an instant when node $C$ was idle, node $A$ finishes its busy period. This busy period is followed by an idle period, $I$, which is exponentially distributed with parameter $\lambda_{A}$. The idle period $I$ ends at time $t=0$. Since it is given that $q_{C}(0)>0$, node $C$ is busy when $I$ terminates.

Our next task is to compute the probability density of the length of the busy period that contains $t=0$, in terms of the known probability density of the length of busy periods of node $C$ (an $M / D / 1$ queue), $f_{X}(x)$. Let $\bar{B}$, an arbitrary busy period of $Q_{C}$ of length $x$, begin at the time $I=I_{0}$, and let $\sigma$ denote the rest of the idle period $I$. Due to the memoryless property of the exponential distribution $I, \sigma$ is exponentially distributed with parameter $\lambda_{A}$. Therefore, the probability that $\bar{B}$ contains $t=0$-given that the length of $\bar{B}$ is $x$-is equal to $\operatorname{Pr}\{\sigma<x\}=$ $\left(1-e^{-\lambda_{A} x}\right)$. Therefore, the probability density of $B$, the busy period that contains $t=0$, is given by

$p_{B}(x)=\left(1-e^{-\lambda_{A} x}\right) f_{X}(x) / \kappa$.

Here, $\kappa=\int_{x=0}^{\infty}\left(1-e^{-\lambda_{A} x}\right) f_{X}(x) d x$ is the normalizing constant. $\kappa$ is computed by noting that $w=\int_{x=0}^{\infty} e^{-\lambda_{A} x} f_{X}(x) d x$ satisfies the functional equation $w=e^{-\left(\lambda_{A}+\lambda_{C}-\lambda_{C} w\right) T}$ [11, page 212], whence $\kappa=1+\frac{W_{0}\left(-\rho_{\mathrm{C}} e^{-\rho_{\mathrm{A}}-\rho_{\mathrm{C}}}\right)}{\rho_{\mathrm{C}}}$.

Finally, the probability density of the residual time $L_{0}$, given that the length of $B$ is $x$, is computed by noting that $\operatorname{Pr}\left\{L_{0} \leq r \mid B=x\right\}=$ $\operatorname{Pr}\{\sigma \geq x-r \mid \sigma \leq x\}$, whence

$p_{L_{0} \mid B}(r \mid x)=\frac{\lambda_{A} e^{-\lambda_{A} x}}{1-e^{-\lambda_{A} x}} e^{\lambda_{A} r}$.

So, $E\left[L_{0} \mid B=x\right]=\frac{x}{1-e^{-\lambda_{A} x}}-\frac{1}{\lambda_{A}}$. Combining with Eq. 34, we obtain

$E\left[L_{0}\right]=\frac{T}{\kappa\left(1-\rho_{\mathrm{C}}\right)}-\frac{T}{\rho_{\mathrm{A}}}$.

Our next goal is to compute $E\left[\sum_{i=1}^{M} X_{i}+Y\right]$. Unlike the previous case, however, $\sum_{i=1}^{M} X_{i}+Y$ is not an integer multiple of $T$. We thus introduce a new variable that will allow us to use the technique presented in section 5.1.

Let $s$ be the smallest integer so that $s T>L_{0}$. We define $\tau=s T-L_{0}$. Due to the memoryless 
property of $\sigma$, it follows that the distribution of $\tau$ is given by

$p_{\tau}(y)=\frac{\lambda_{A} e^{-\lambda_{A} y}}{1-e^{-\rho_{\mathrm{A}}}}$,

where $y \in[0, T]$. It follows then

$E[\tau]=T / \rho_{\mathrm{A}}-T /\left(e^{\rho_{\mathrm{A}}}-1\right)$.

We now note that $\sum_{i=1}^{M} X_{i}+Y-\tau$ is an integer multiple of $T$. This is because, rewriting Eq. 30,

$\sum_{i=1}^{M} X_{i}+Y-\tau=N T-\sum_{i=1}^{M} L_{i}-T-\left(L_{0}+\tau\right)$,

and all the terms in the R.H.S of Eq. 39 are integer multiples of $T$. So, we can write $\sum_{i=1}^{M} X_{i}+Y=\tau+(K-1) T$.

To compute $E[K]$, we will apply the same technique that we used in section 5.1. We construct a new time axis $t^{\prime}$ from $t$ by removing the busy periods $L_{i}(i=1,2, \ldots)$. This new axis is shown in Fig. 6. The only difference is that we choose $t^{\prime}$ in such a way that the point of time $t=L_{0}$ is mapped to $t^{\prime}=-\tau$. As we explained in section 5.1 , the $m$-th busy period on $t^{\prime}$ axis is represented by a point occurrence at time $t^{\prime}=\sum_{i=1}^{m} X_{i}-\tau$, and these occurrences constitute a Poisson process with intensity $\lambda_{C}$. We again denote this process by $\mathcal{P}$. The interval $[(N-1) T, N T]$ on $t$ axis is mapped to $[(K-1) T, K T]$ on $t^{\prime}$ axis. $K$ is again characterized to be the smallest positive integer so that the interval $[(K-1) T, K T]$ on $t^{\prime}$ axis has no arrival of the process $\mathcal{P}$.

The probability distribution of $K$ remains as before; namely,

$$
\begin{aligned}
\operatorname{Pr}(K=k) & =\left(1-e^{-\rho_{\mathrm{C}}}\right)^{k-1} e^{-\rho_{\mathrm{C}}}, \text { and } \\
E[K] & =e^{\rho_{\mathrm{C}}} .
\end{aligned}
$$

To compute $E[M]$, we count the number of arrivals in each sub-interval. Define $\nu_{0}$ to be the number of arrivals of $\mathcal{P}$ during the interval $[-\tau, 0]$, and $\nu_{j}(j \geq 1)$ to be the number of arrivals of $\mathcal{P}$ during the interval $[(j-1) T, j T]$ on $t^{\prime}$ axis. Then, $M$, the total number of arrivals of $\mathcal{P}$ during the interval $[-\tau, K T]$ is the sum of $\nu_{j}$ 's:

$M=\sum_{j=0}^{K} \nu_{j}=\sum_{j=0}^{K-1} \nu_{j}$ since $\nu_{K}=0$ by the definition of $K . \quad \nu_{j}(j=$ $1,2, \ldots, K-1)$ are i.i.d and independent of $\tau$. However, $\nu_{0}$, depends on $\tau$. Also note that the possibility $\nu_{0}=0$ is not excluded. We compute the expectation of $\nu_{0}$ separately by conditioning on $\tau$. Since $\mathcal{P}$ is a Poisson process, $E\left[\nu_{0} \mid \tau\right]=$ $\lambda_{C} \tau$, whence, $E\left[\nu_{0}\right]=\lambda_{C} E[\tau]=\frac{\rho_{\mathrm{C}}}{\rho_{\mathrm{A}}}-\frac{\rho_{\mathrm{C}}}{e^{\rho_{\mathrm{A}}-1}}$. Here, we substituted $E[\tau]$ from Eq. 38. Therefore,

$$
\begin{aligned}
E[M] & =E\left[\nu_{0}\right]+(E[K]-1) E[\nu \mid \nu>0] \\
& =\frac{\rho_{\mathrm{C}}}{\rho_{\mathrm{A}}}-\frac{\rho_{\mathrm{C}}}{e^{\rho_{\mathrm{A}}}-1}+\rho_{\mathrm{C}} e^{\rho_{\mathrm{C}}} .
\end{aligned}
$$

Now we substitute $E[K]$ from Eq. 41 and $E[M]$ from Eq. 44 into 33, and obtain the desired expectation:

$$
\begin{aligned}
& E\left[N \mid q_{C}(0)>0\right] \\
= & \frac{e^{\rho_{\mathrm{A}}+\rho_{\mathrm{C}}}-e^{\rho_{\mathrm{C}}}-1}{\left(e^{\rho_{\mathrm{A}}}-1\right)\left(1-\rho_{\mathrm{C}}\right)}+\frac{\rho_{\mathrm{A}}+\kappa \rho_{\mathrm{C}}}{\kappa \rho_{\mathrm{A}}\left(1-\rho_{\mathrm{C}}\right)} .
\end{aligned}
$$

This proves Eq. 6.

The following quantity will be used in section 5.3. Let $W$ be the amount of time node $A$ transmits and node $C$ does not during the transmission attempts of this packet. Then,

$W=\left[\sum_{i=1}^{M} X_{i}+Y\right]+T$.

It follows that

$$
\begin{aligned}
& E\left[W \mid q_{C}(0)>0\right] \\
= & E\left[\sum_{i=1}^{M} X_{i}+Y \mid q_{C}(0)>0\right]+T \\
= & T\left[\frac{1}{\rho_{\mathrm{A}}}-\frac{1}{e^{\rho_{\mathrm{A}}}-1}+e^{\rho_{\mathrm{C}}}\right] .
\end{aligned}
$$

5.3. Derivation of $\operatorname{Pr}\left\{q_{C}(0)>0\right\}$

In this section, we derive Eq. 7, the probability that node $C$ is transmitting at the time node $A$ starts transmitting a fresh packet, i.e. $q_{C}(0)>0$.

We first observe that the event $q_{C}(0)>0$ occurs with nonzero probability if and only if an arrival to node $A$ finds $Q_{A}$ empty and $Q_{C}$ busy. Clearly, $q_{C}(0)>0$ for all such arrivals, since the packet is transmitted immediately. It is also clear that $q_{C}(0)=0$ for the arrivals that find both $Q_{A}$ and $Q_{C}$ empty. On the other hand, if an arrival finds $Q_{A}$ nonempty - i.e. it is queued - then 
with probability 1 , node $C$ is empty at the time this packet is transmitted; otherwise, the previous packet sent by node $A$ would not have been transmitted successfully. So,

$\operatorname{Pr}\left\{q_{C}(0)>0\right\}=\operatorname{Pr}\{$ An arrival to node $A$ finds

$Q_{A}$ empty and $Q_{C}$ nonempty $\}$.

However, by the PASTA (Poisson Arrivals See Time Average) property, an arrival to node $A$ finds the system is at its steady-state distribution. Thus,

$\operatorname{Pr}\left\{q_{C}(0)>0\right\}=\operatorname{Pr}\left\{q_{A}=0, q_{C}>0\right\}$.

We will derive Eq. 49 by computing $\operatorname{Pr}\left\{q_{A}=\right.$ $\left.0, q_{C}=0\right\}$ using Little's law. We observe that the event $q_{A}=0, q_{C}=0$ is equivalent to that the system consists of $Q_{A}$ and $Q_{C}$ is empty. So, we can find $\operatorname{Pr}\left\{q_{A}=0, q_{C}=0\right\}$ by applying Little's law to the whole system. The utilization of the system is given by:

System Utilization $=$ fraction of time node $C$ transmits + fraction of time node $A$ transmits and node $C$ does not.

Let $W$ denote the amount of time node $A$ transmits and node $C$ does not during the transmission attempts of a packet. We can then write the System Utilization:

$\bar{\rho}_{\text {sys }}=\lambda_{C} T+\lambda_{A} E[W]$,

and the probability that the system is empty

$\operatorname{Pr}\left\{q_{A}=0, q_{C}=0\right\}=1-\bar{\rho}_{\text {sys }}$.

We compute $E[W]$ by conditioning. We distinguish between the cases when $q_{C}(0)=0$ and $q_{C}(0)>0$. The conditional expectations are given by Eq. 29 and Eq. 48. So,

$$
\begin{aligned}
& E[W] \\
= & E\left[W \mid q_{C}(0)>0\right] \cdot \operatorname{Pr}\left\{q_{C}(0)>0\right\} \\
+ & E\left[W \mid q_{C}(0)=0\right]\left(1-\operatorname{Pr}\left\{q_{C}(0)>0\right\}\right) \\
= & T\left[\left(\frac{1}{\rho_{\mathrm{A}}}-\frac{1}{e^{\rho_{\mathrm{A}}}-1}\right) \cdot \operatorname{Pr}\left\{q_{A}=0, q_{C}>0\right\}\right. \\
+ & \left.e^{\rho_{\mathrm{C}}}\right]
\end{aligned}
$$

The substitution of $\operatorname{Pr}\left\{q_{C}(0)>0\right\}$ is made using Eq. 49.

Various probabilities are related by the fundamental relation:

$$
\begin{aligned}
\operatorname{Pr}\left\{q_{A}=0, q_{C}>0\right\} & =\operatorname{Pr}\left\{q_{A}=0\right\} \\
& -\operatorname{Pr}\left\{q_{A}=0, q_{C}=0\right\} .
\end{aligned}
$$

Substituting $\operatorname{Pr}\left\{q_{A}=0\right\}$ from Eq. 9, $\operatorname{Pr}\left\{q_{A}=\right.$ $\left.0, q_{C}=0\right\}$ from Eq. 51 and $E[W]$ from Eq. 54 in Equation 55, we get our desired expression

$$
\begin{aligned}
& \operatorname{Pr}\left\{q_{C}(0)>0\right\}=\operatorname{Pr}\left\{q_{A}=0, q_{C}>0\right\} \\
= & \frac{\left(e^{\rho_{\mathrm{A}}}-1\right)\left(e^{\rho_{\mathrm{C}}}-P_{\text {coll }}-e^{\rho_{\mathrm{C}}} P_{\text {coll }}\right)}{1-P_{\text {coll }}} .
\end{aligned}
$$

This proves Eq. 7.

\section{Simulation Results}

In this section we present simulation results that verify the correctness of our analysis as well as its ability to predict the performance of IEEE 802.11 networks.

\subsection{Validation of the Analysis}

In order to validate our analysis, we have developed a discrete event simulator in Matlab [30] that simulates the elementary topology shown in Fig. 2(a) and make exactly the same modeling assumptions as those described in section 3: namely, the packet arrivals at the nodes are independent Poisson processes with rate $\lambda$, each frame takes one unit time to transmit, unsuccessful frames are retransmitted immediately, and maximum retransmission per packet is unlimited. The simulation is run for 100,000 unit time. Since there is no modeling mismatch, we expect essentially an exact match between the simulation and the theory.

This is indeed the case as shown in Figs. 8 and 9 . Fig. 8 plots the probability of packet collision (the Packet Error Rate), while Fig. 9 plots the mean system time of a packet. Both figures compare the analytical results with those obtained by simulation. From the plots, it is clear that the match between the simulation and analytical results is excellent, which validates the correctness of our analytical results. 


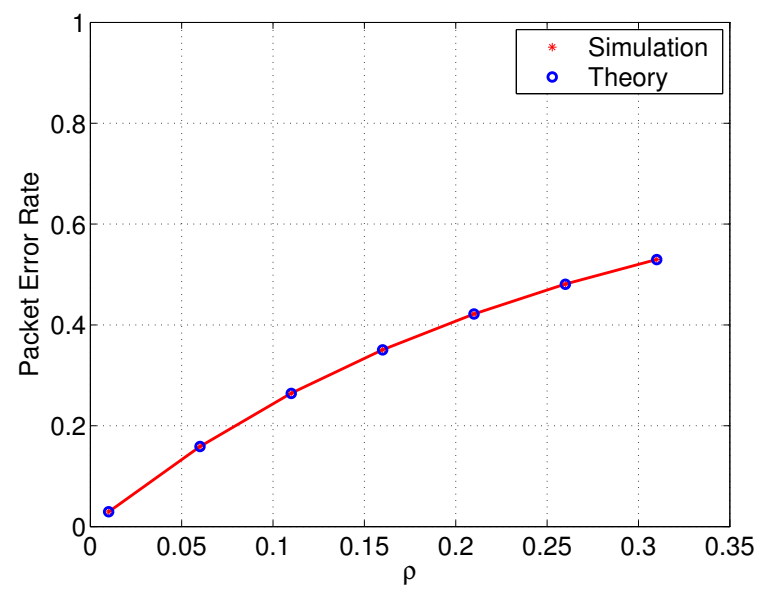

Figure 8. Simulation vs. Theory: Probability of packet collision.

\subsection{Simulation of IEEE 802.11 network}

Verifying the applicability of our analytical models to real systems is our next task. In order to do so, we have created a simulation environment based on the $n s$ simulator [26], which simulates wireless networks based on IEEE 802.11 standard. Table 1 summarizes the main parameter settings for the simulator. The values used for retry limits and propagation model are default. RTS threshold is set to 3000 so that nodes do not use RTS / CTS handshake.

\subsubsection{Elementary Topology}

We start by describing simulation outcomes for the elementary topology shown in Fig. 2(a).

Probability of packet collision: Fig. 10 compares the probability of packet collision resulted in NS simulation, and the theoretical prediction. From the plot, it is clear that the simulation and the analysis match very well. Small discrepancy is observed only at a very high traffic load $(\rho \approx 0.4$ is the saturation point), where some of our modeling assumptions are no longer valid. We also point out that the probability of packet collision is already $50 \%$ in this area. So, a practical network may anyway not be able to work at such

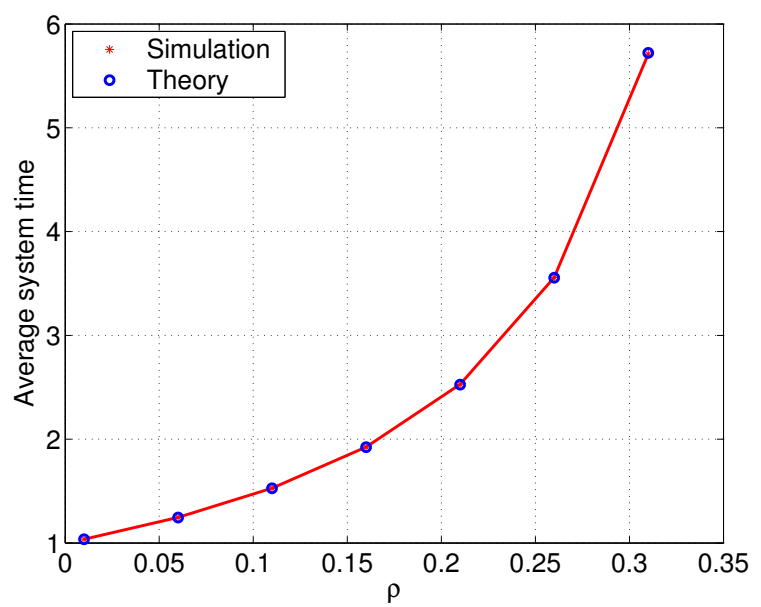

Figure 9. Simulation vs. Theory: Average delay seen by the packets at node $A$.

high traffic loads.

Average system time: Next, we compare the simulation and theoretical results for the average system time, shown in Fig. 11. The $y$-axis is normalized by the packet transmission time $T$. It is clear that the theoretical prediction matches well with the simulation results, especially at lower values of traffic load. At higher values of traffic load, where probability of collision is nearly $50 \%$ (cf. Fig. 10), some discrepancy arises due to nonzero backoff intervals.

Maximum throughput: Finally, we consider the maximum throughput of this network. Fig. 12 shows the average system time, normalized by the packet transmission time $T$, versus the traffic load. The simulation clearly indicates that the queue of node $A$ saturates around a load of $\rho=0.4$. The theoretical prediction of the maximum throughput, derived in section 4.2 , is $\rho^{*}=0.401$ which agrees very well with the simulation result.

\subsubsection{Linear Topology}

Now we describe simulation outcomes for the general linear network. The topology is shown in Fig. 4 , where we used 15 pairs of nodes: $\left(A_{0}, B_{0}\right)$ 
Table 1

Key NS parameters.

\begin{tabular}{|l|l|}
\hline Routing protocol & DumbAgent \\
\hline Propagation model & TwoRayGround \\
\hline Data rate & $1 \mathrm{Mb} / \mathrm{s}$ \\
\hline Carrier sense threshold & $2.5 \times 10^{-10}$ \\
\hline Receiving range Threshold & $2.5 \times 10^{-10}$ \\
\hline Packet size & 2000 Byte \\
\hline Short retry limit & 7 \\
\hline Long retry limit & 4 \\
\hline RTS Threshold & 3000 \\
\hline
\end{tabular}

to $\left(A_{14}, B_{14}\right)$. Exogenous arrivals at each node $A_{i}$ follow independent Poisson processes, each with a common rate $\lambda$ (hence, by definition, $\rho=\lambda T$ ).

Probability of packet collision: Fig. 13 compares the analytical and ns simulation results for probability of collision at nodes $A_{1}, A_{7}$ and $A_{14}$. The figure clearly shows that our analytical expression is asymptotically exact at low load. Moreover, the analysis clearly captures the behavior of the curves, although discrepancies are observed when the probability of collision is high.

It is interesting to observe that as we go further to the left (cf. Fig. 4), the probability of collision becomes very high even at relatively low load. This is perhaps surprising since packets of node $A_{i}$ are only affected by the transmissions of node $A_{i-1}$. An intuitive reason is as follows: due to the transmissions at node $A_{0}$, node $A_{1}$ 's packets collide, which are retransmitted. These retransmissions cause further packet collisions at node $A_{2}$. So, node $A_{2}$ 's packets experience a higher probability of packet collision, which in turn affects node $A_{3}$; and so on. Therefore, the impact of hidden nodes is cumulative in this topology.

Maximum throughput: The nodes in the linear network can achieve different maximum throughput. In particular, when the common exogenous load value, $\rho$, is increased, the leftmost node saturates first, due to the cumulative effect explained above. In Table 2, we show the maximum throughput observed at nodes $A_{1}, A_{7}$ and $A_{14}$ in the simulation as well as the theoretical predictions.

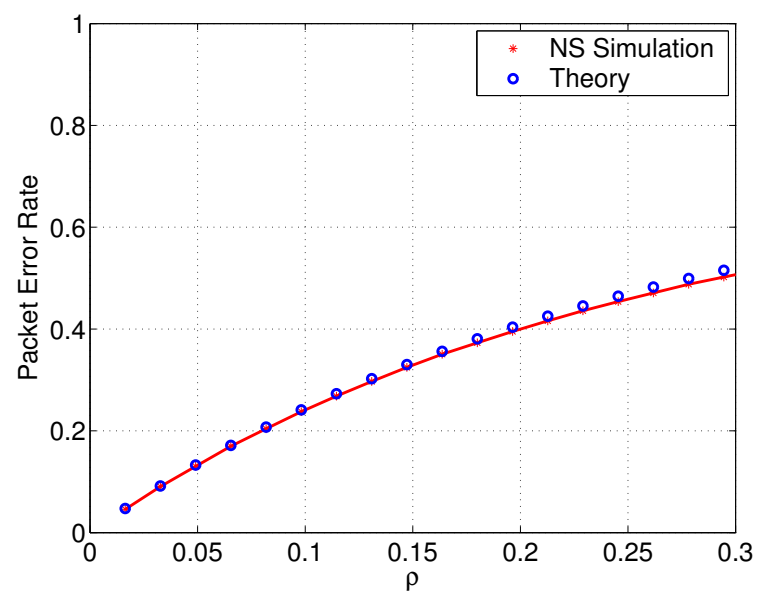

Figure 10. Probability of packet collision: Comparison between NS simulation and theory.

Table 2

Maximum throughput in the linear network.

\begin{tabular}{|c|c|c|}
\hline & Theory & Simulation \\
\hline$A_{1}$ & 0.401 & 0.400 \\
\hline$A_{7}$ & 0.160 & 0.185 \\
\hline$A_{14}$ & 0.140 & 0.160 \\
\hline
\end{tabular}

We find that the theoretical predictions are lower than simulation outcomes. The discrepancy occurs due to the fact that our theoretical results for the general linear network are based on low load assumption, so they are less accurate near the saturation point. Nevertheless, the theory does predict the right order of magnitude - in fact, it provides a lower estimate - which is often sufficient for many purposes.

\section{Conclusion}

In this paper, we have quantified the impact of hidden nodes on the performance of linear wireless networks. We have performed an analysis based on a model that closely follows the IEEE 802.11 protocol, taking into consideration the ef- 


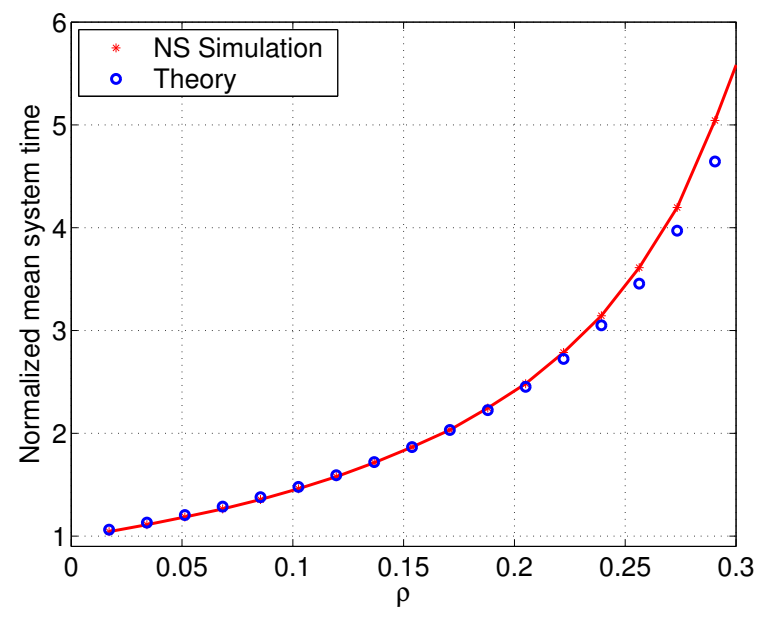

Figure 11. Average system time: NS simulation vs. theory.

fects of queuing and retransmissions at each node.

In order to make the analysis tractable, we have decomposed the general network into elementary 4-node topologies. For these topologies and based on our statistical assumptions, we have derived exact expressions for the probability of packet collision, maximum throughput and mean packet delay. We then generalized these results to the case of general linear wireless networks. In particular, we developed a simple iterative procedure that allows to approximate the packet collision probability and maximum throughput at each node of a linear wireless network.

We made use of simulation to verify the correctness of our analysis as well as its applicability to predict the performance of IEEE 802.11 networks. The analytical results were verified by a Matlab based discrete event simulation, whereas we used the IEEE 802.11 standard NS simulator to verify the merits of the analytical predictions. The Matlab simulation results matched exactly with the analytical predictions, thereby validating our analysis. The NS simulations showed that our analytical results provide high quality predictions of network performance; slight discrepancy

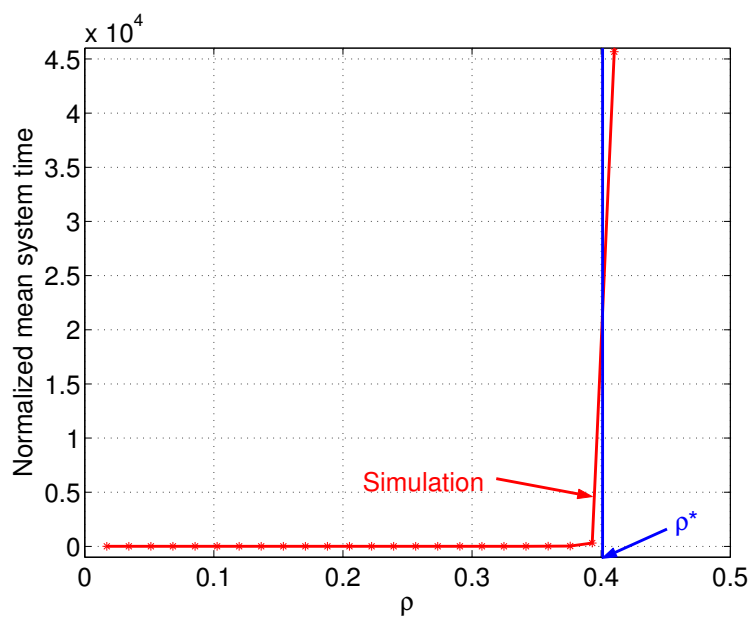

Figure 12. Maximum throughput: NS simulation. Theoretical prediction is $\rho^{*}=0.401$.

was observed only at traffic load where the probability of packet collision is already very high. For the case of the general linear network, we also observed and discussed an interesting propagation effect, whereby some of the nodes saturate at traffic load values as low as $\rho \approx 0.15$, even though each node is directly affected by only one other node.

Although our analysis focused on IEEE 802.11 networks, it can also be used for predicting the performance of other types of wireless networks, such as sensor networks. Since sensor networks often implement CSMA-based protocols (see e.g. [31, 32]), the scenarios described and analyzed in this paper could be especially useful in the design and evaluation of these networks. In this respect, the extension of our analysis to general topologies represents a very interesting research area open for future work.

\section{REFERENCES}

1. Wireless LAN Medium Access Control (MAC) and Physical Layer (PHY) Specification, ANSI/IEEE Std. 802.11, 1999. 


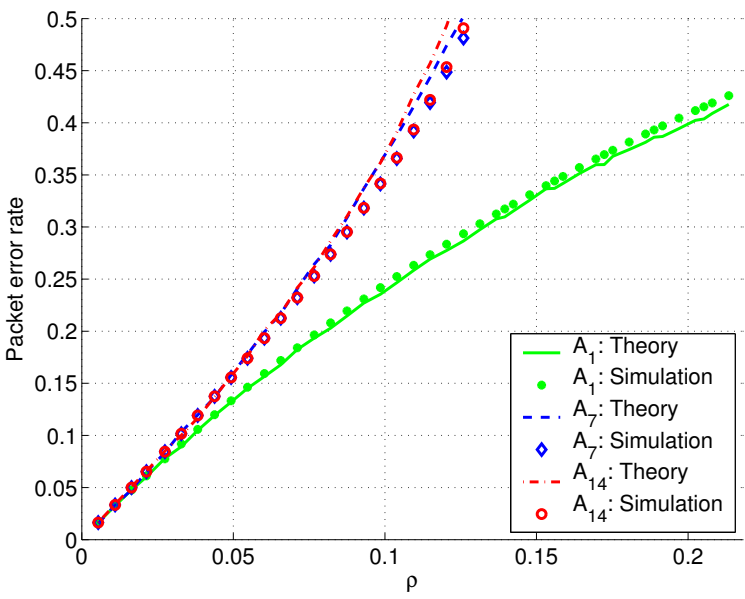

Figure 13. Probability of packet collision: Comparison between NS simulation and theory.

2. L. Kleinrock and F. A. Tobagi, "Packet switching in radio channels: Part 1 - Carrier Sense Multiple-Access modes and their throughput-delay characteristics," IEEE Transactions on Communications, vol. COM-23, no. 12, pp. 1400-1416, 1975.

3. M. W. Ritter, "The future of WLAN," $A C M$ Queue: Tomorrow's Computing Today, vol. 1, no. 3, May 2003.

4. S. Ray, J. Carruthers, and D. Starobinski, "RTS/CTS-induced congestion in adhoc wireless LANs," in Proceedings of IEEE $W C N C$, New Orleans, March 2003, pp. 15161521.

5. K. Xu, M. Gerla, and S. Bae, "How effective is the IEEE 802.11 RTS/CTS handshake in ad hoc networks?" in IEEE Globecom. IEEE, 2002.

6. S. Choi and J. del Prado, "802.11g CP: A solution for IEEE $802.11 \mathrm{~g}$ and $802.11 \mathrm{~b}$ interworking," in Proceedings of IEEE VTC'03Spring, Jeju, Korea, April 2003.

7. Z. J. Haas, J. Deng, B. Liang, P. Papadimitratos, and S. Sajama, "Wireless ad hoc networks," in Encyclopedia of Telecommunica- tions, J. G. Proakis, Ed. John Wiley, 2002.

8. S. Venkatesh, N. Arcolano, and D. Starobinski, "Influence propagation in wireless ad-hoc and sensor networks," preprint.

9. PC4500/PC4800 Developer's Reference Manual, Aironet Wireless Communications, 1997, document number: 710-004247, Revision: B1.

10. F. A. Tobagi and L. Kleinrock, "Packet switching in radio channels: Part 2 - the hidden node problem in carrier sense multiple access modes and the busy tone solution," IEEE Transactions on Communications, vol. COM23, no. 12, pp. 1417-1433, 1975.

11. L. Kleinrock, Queuing Systems: Volume 1. John Wiley \& Sons, 1975.

12. Z. J. Haas and J. Deng, "Dual busy tone multiple access (DBTMA) - a multiple access control scheme for ad hoc networks," vol. 50, no. 6, pp. 977-985, June 2002.

13. C. L. Fullmer and J. Garcia-Luna-Aceves, "Floor acquisition multiple access (FAMA) for packet-radio networks," in Proceedings of SIGCOMM '95. ACM, 1995.

14. —— "Solutions to hidden terminal problems in wireless networks," in Proceedings of SIGCOMM '97. ACM, 1997.

15. A. Zahedi and K. Pahlavan, "Natural hidden terminal and the performance of the wireless LANs," in Universal Personal Communications Record, 1997.

16. G. Bianchi, "Performance analysis of the IEEE 802.11 distributed coordination function," IEEE Journal on Selected Areas in Communications, Wireless series, vol. 18, no. 3, March 2000.

17. L. Wu and P. K. Varshney, "Performance analysis of CSMA and BTMA protocols in multihop networks (i). single channel case," Information Sciences, vol. 120, pp. 159-177, November 1999.

18. —_ "Performance analysis of CSMA and BTMA protocols in multihop networks (ii). multiple channel case," Information Sciences, vol. 120, pp. 179-195, November 1999.

19. W. M. Moh, D. Yao, and K. Makki, "Analyzing the hidden-terminal effects and multimedia support for wireless LAN," Computer 
Communications (Elsevier), vol. 23, no. 10, pp. 998-1013, May 2000.

20. S. Khurana, A. Kahol, S. K. S. Gupta, and P. K. Srimani, "Performance evaluation of distributed co-ordination function for IEEE 802.11 wireless LAN protocols in presence of mobile and hidden terminals," in Proceedings of the 7th International Symposium on Modeling, Analysis and Simulation of Computer and Telecommunication Systems. College Park, Maryland, USA: IEEE Computer Society, 24-28 October 1999.

21. M. Sidi and A. Segall, "Two interfering queues in packet-radio networks," IEEE Transactions on Communications, vol. COM31, no. 1, pp. 123-129, Jan. 1983.

22. H. Takagi and L. Kleinrock, "Mean packet queuing delay in a buffered two-user CSMA/CD system," IEEE Transactions on Communications, vol. COM-33, no. 10, October 1985.

23. J. Mišic and V. B. Mišic, "Modeling bluetooth piconet performance," IEEE Communication Letters, vol. 7, no. 1, pp. 18-20, Jan. 2003.

24. I. Papanikos and M. Logothetis, "A study on dynamic load balance for IEEE 802.11b wireless LAN," in Proceedings of the 8th International Conference on Advances in Communication 8 Control, COMCON 8, Rethymna, Crete/Greece, June 2001.

25. D. Gross and C. M. Harris, Queuing Theory (3rd edition). John Wiley \& Sons, 1998.

26. [Online]. Available: http://www.isi.edu/ nsnam

27. S. Ray, J. B. Carruthers, and D. Starobinski, "Evaluation of the masked node problem in ad-hoc wireless LANs," IEEE Transactions on Mobile Computing, In press.

28. R. M. Corless, G. H. Gonnet, D. E. G. Hare, D. J. Jeffrey, and D. E. Knuth, "On the Lambert W function," Advances in Computational Mathematics, vol. 5, pp. 329-359, 1996.

29. D. Bertsekas and R. Gallager, Data Networks, 2nd ed. Prentice Hall, 1991.

30. [Online]. Available: http://www.mathworks. com

31. IEEE Standard for Information TechnologyPart 15.4: Wireless Medium Access Con- trol (MAC) and Physical Layer (PHY) specifications for Low Rate Wireless Personal Area Networks (LR-WPANS), ANSI/IEEE Std. 802.15.4, 2003.

32. A. Woo and D. Culler, "A transmission control scheme for media access in sensor networks," in ACM International Conference on Mobile Computing and Networking (MobiCom). ACM, 2001. 


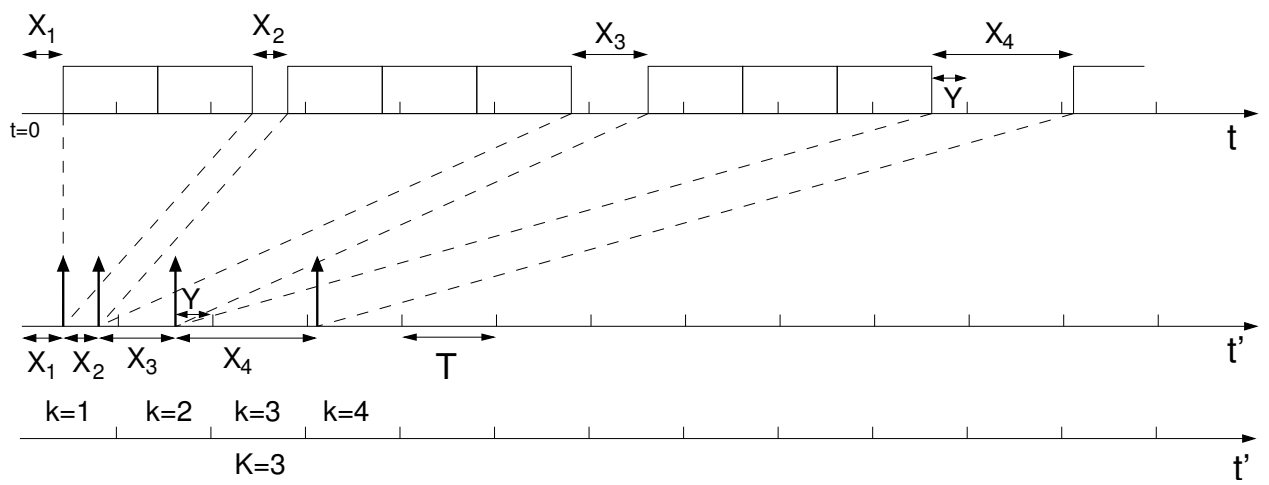

Figure 5. Description of the notation for the case $q_{C}(0)=0$.

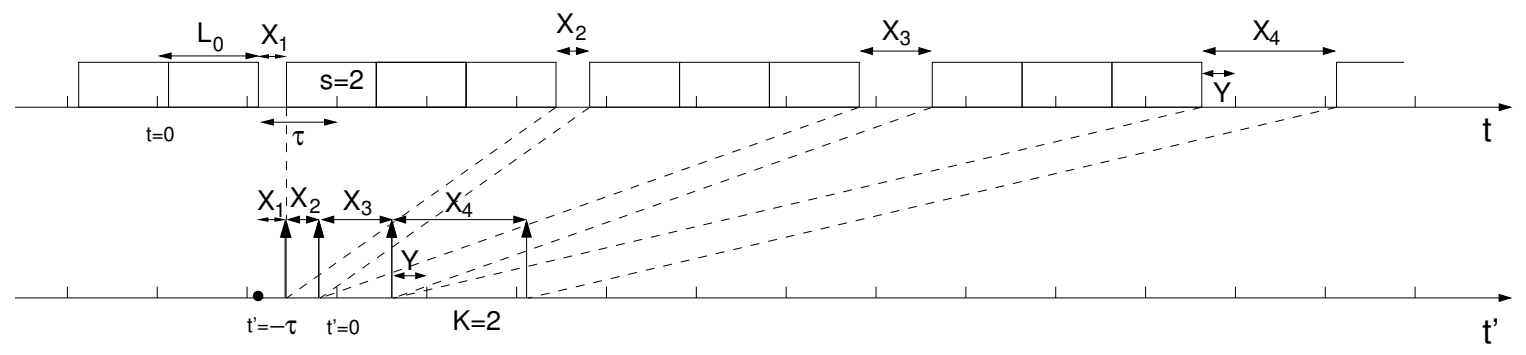

Figure 6. Description of the notation for the case $q_{C}(0)>0$.

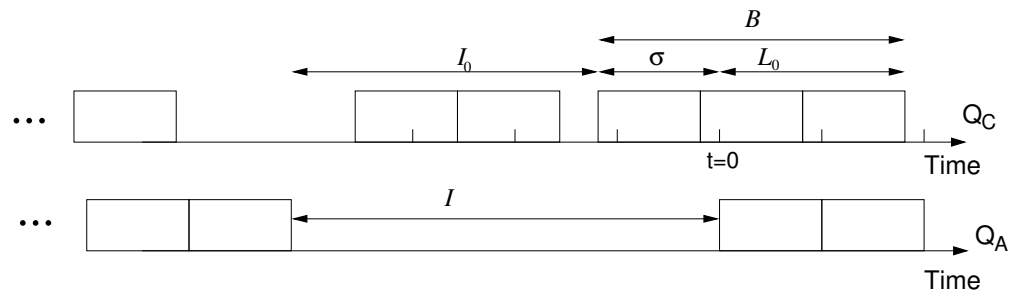

Figure 7. A typical sample path for the case $q_{C}(0)>0$. 